\title{
Albumin is required to support the acrosome reaction but not capacitation in mouse spermatozoa in vitro
}

\author{
Lynn R. Fraser
}

Department of Anatomy and Human Biology, King's College, Strand, London WC2R 2LS, U.K.

\begin{abstract}
Summary. Albumin was required specifically for penetration of the zona pellucida ( $<10 \%$ of eggs fertilized in the absence of albumin), but was not required for capacitation. A similar rate of capacitation was observed in the presence of albumin at concentrations ranging from 30 to $1 \mathrm{mg} / \mathrm{ml}$, while a slightly slower rate was observed in the presence of 0.25 and $0.1 \mathrm{mg}$ albumin $/ \mathrm{ml}$. In the absence of albumin, capacitation occurred at a rate which lagged behind that of the albumin-incubated counterparts by about $30 \mathrm{~min}$; once capacitated, the addition of albumin promoted rapid sperm penetration. In albumin-free media ( \pm the macromolecule PVA), sperm motility was frequently reduced, with fewer cells exhibiting hyperactivated motility, but improvements were observed after introduction of albumin. Acrosome loss was significantly lower in the absence of albumin, but within $5 \mathrm{~min}$ of its addition at concentrations ranging from 30 to $0.1 \mathrm{mg} / \mathrm{ml}$ to capacitated sperm suspensions, acrosome loss was stimulated and reached levels similar to those seen in control samples. Therefore, albumin can trigger the acrosome reaction in capacitated spermatozoa. It appears to act by assisting in the removal of a surface-associated inhibitory component, the presence of which stabilizes the sperm membranes and inhibits the acrosome reaction.
\end{abstract}

\section{Introduction}

Successful fertilization in vitro usually requires the presence of a proteinaceous macromolecule, the most common one used being albumin (Miyamoto \& Chang, 1973; Hoppe \& Whitten, 1974; Bavister \& Yanagimachi, 1977; Quinn, Stanger \& Whittingham, 1982). Although the specific mechanisms whereby albumin exerts its effects are not clearly defined, Lui, Cornett \& Meizel (1977) have reported that albumin is required for induction of the acrosome reaction in hamster spermatozoa and that the fatty acid content of albumin can either enhance or inhibit acrosome loss (Lui \& Meizel, 1977). Albumin is also very effective in maintaining sperm motility during extended incubation in vitro (see for example Lui et al., 1977; Suter, Chow \& Martin, 1979; Harrison, Dott \& Foster, 1982). Since motility is required for fertilization of eggs, at least those with intact investments, this may represent another facet of the albumin requirement.

Using a well characterized in-vitro fertilization system (Fraser, 1983a, 1984), the present study was undertaken to examine more closely the nature of an albumin requirement in mouse spermatozoa for capacitation, fertilization, maintenance of motility and induction of the acrosome reaction.

\section{Materials and Methods}

Media. A modified Tyrode's solution (calculated osmolality 318 mosmol; Fraser, 1983b) was used in all experiments. The standard medium contained albumin (crystalline BSA at $4 \mathrm{mg} / \mathrm{ml}$; Armour, Eastbourne, Sussex, U.K.); essentially fatty acid-free BSA (faf BSA; Sigma, Poole, Dorset, U.K.):21:23AM 
was also used in a few experiments. In addition, BSA-free medium and medium lacking BSA but containing polyvinyl alcohol (PVA; Sigma) were used. PVA has been shown to maintain gamete viability but not to support the acrosome reaction (Bavister, 1981). In one series of experiments, hyperosmolal media ( 368 and 418 mosmol) were prepared by increasing $\mathrm{NaCl}$ from $124.54 \mathrm{mM}$ to 149.54 and $174.54 \mathrm{~mm}$, respectively.

In-vitro fertilization. Mature TO female mice ( $>8$ weeks) were induced to superovulate by i.p. injections of 7.5 i.u. PMSG (Gestyl: Organon, Morden, Surrey, U.K.) and, $48-54$ h later, 5 i.u. hCG (Pregnyl:Organon). All eggs were recovered $14 \mathrm{~h}$ after hCG injection; when sperm suspensions in a single experiment were assessed after various lengths of preincubation, hCG was injected asynchronously. Except where noted, cumulus-intact eggs were released from oviducts directly into sperm suspensions. Cumulus-free eggs were prepared by treatment with hyaluronidase and zona-free eggs by exposure of the former to pronase (see Fraser, 1983a). For zona removal, pronase was prepared in BSA-containing medium, with eggs being extensively washed in BSA-free medium subsequently, or in PVA-containing medium, again with similar washing. The inclusion of a macromolecule such as PVA reduces the possibility that zona-free eggs will adhere irreversibly to the dish; in a protein-free medium, these eggs are very sticky and difficult to work with. Results with both treatments were similar and have been combined.

Epididymal sperm suspensions were prepared by releasing the contents of 1 cauda epididymidis from each of 2 mature ( $>8$ weeks) TO male mice into $1 \mathrm{ml}$ medium; in most experiments 1 suspension was prepared in BSA-containing medium and a second in albumin-free ( \pm PVA) medium. Except as noted, suspensions were preincubated for $120 \mathrm{~min}$. Samples were diluted approximately 10 -fold to give a concentraton of $1-2 \times 10^{6}$ cells $/ \mathrm{ml}$ and eggs were released into $300 \mu \mathrm{l}$ droplets of these suspensions.

All incubations were carried out in $30 \mathrm{~mm}$ plastic culture dishes (Sterilin, Teddington, Middx., U.K.) and the droplets of medium were overlaid with autoclaved liquid paraffin (Boots, Nottingham, U.K.). The gas phase used throughout was $5 \% \mathrm{CO}_{2}-5 \% \mathrm{O}_{2}-90 \% \mathrm{~N}_{2}$.

Washing of sperm suspensions. Sperm suspensions were washed gently by mixing 1 volume of suspension with 2 volumes of the same medium used for sperm release and centrifuging at $750 \mathrm{~g}$ (room temperature) for $5 \mathrm{~min}$. The supernatant was removed and the pellet resuspended to the original volume, again in the same medium used to prepare the suspension.

Preparation of sperm supernatants. One volume of sperm suspension was mixed with 1 volume of medium (dilution factor of $1 / 2$ ) and centrifuged at $11600 \mathrm{~g}$ (room temperature) for $4 \mathrm{~min}$. The resulting cell-free supernatant was used to dilute preincubated sperm suspensions.

Assessment of acrosome loss. After preincubation for 30 or $120 \mathrm{~min}$, aliquants of sperm suspensions were diluted 10 -fold, incubated for $5 \mathrm{~min}$ and then subjected to filtration on short columns prepared in Pasteur pipettes (Fraser \& Quinn, 1981) to select motile, potentially fertilizing spermatozoa for assessment. Preliminary studies indicated that a macromolecular component (PVA) in the medium and a column made of Sephadex G-25 (Fraser \& Quinn, 1981), rather than glass beads (Fraser, 1983b), prevented excessive sticking of cells during filtration which might distort the results. This problem does not arise when BSA is included in the medium. The filtrates were fixed in formalin, slides were prepared (Fraser, 1983b) and at least 100 cells in each sample were evaluated for the presence or absence of an acrosome.

Routine assessments. After 65-70 min, eggs were transferred from the sperm suspensions to small droplets of fresh medium; at $75 \mathrm{~min}$, eggs were fixed by adding an excess of neutral buffered formalin ( $4 \%$ formaldehyde). After staining and mounting as described by Fraser (1983a), eggs were assessed; they were considered to be fertilized if they had resumed the second meiotic division and contained a decondensing sperm head. To determine the rate of sperm penetration, the proportion of fertilized eggs exhibiting maximal nuclear development, i.e. eggs at the telophase- 
second polar body stage and possessing a fully decondensed sperm head (see Fraser, 1983a), was calculated.

Preincubated and diluted sperm suspensions were assessed for the proportion of motile cells and for expression of hyperactivated motility, a specific motility pattern associated with zona penetration (Fraser, 1981).

Cochran's test for the combination of $2 \times 2$ contingency tables (Snedecor \& Cochran, 1967) was used to analyse results.

\section{Results}

Series $I:$ is albumin required for fertilization?

Unwashed suspensions. Sperm suspensions were prepared in BSA-containing and BSA-free media. After preincubation for $120 \mathrm{~min}$, aliquants were diluted in the same medium used for preincubation and assessed for their ability to fertilize cumulus-intact, cumulus-free and zona-free eggs. The effect of releasing cumulus masses into BSA-free medium to reduce oviducal fluid components before transfer to BSA-free sperm suspensions was also examined. Finally, an aliquant of suspension preincubated in BSA-free medium was diluted in BSA-containing medium. Four replicate experiments were performed $(\mathrm{N}=4)$.

Table 1. In-vitro fertilizing ability of mouse sperm suspensions preincubated for $120 \mathrm{~min}$ in the presence or absence of BSA and then tested with cumulus-intact, cumulus-free and zonal-free eggs

\begin{tabular}{|c|c|c|c|c|c|c|c|}
\hline \multicolumn{2}{|c|}{ Medium } & \multicolumn{2}{|c|}{ Eggs } & \multicolumn{2}{|c|}{ Eggs fertilized } & \multirow{2}{*}{$\begin{array}{l}\text { Maximal nuclear } \\
\text { development } \dagger, \%\end{array}$} & \multirow{2}{*}{$\begin{array}{l}\text { Polyspermic } \\
\text { eggs, } \%\end{array}$} \\
\hline Preincubation & Fertilization & \pm Cumulus & \pm Zona & No., $\%$ & (range) & & \\
\hline$+\mathrm{BSA}$ & $+\mathrm{BSA}$ & $\begin{array}{l}+ \\
- \\
-\end{array}$ & $\begin{array}{l}+ \\
+ \\
-\end{array}$ & $\begin{array}{l}79 / 83,95 \cdot 2 \\
49 / 59,83 \cdot 1 \\
32 / 32,100\end{array}$ & $\begin{array}{l}(87-100) \\
(71-100)\end{array}$ & $\begin{array}{r}100 \\
88 \cdot 6 \\
100\end{array}$ & $\begin{array}{c}- \\
- \\
50 \cdot 0\end{array}$ \\
\hline - BSA & - BSA & $\begin{array}{l}+ \\
+\ddagger \\
- \\
-\end{array}$ & $\begin{array}{l}+ \\
+ \\
+ \\
-\end{array}$ & $\begin{array}{c}33 / 65,50 \cdot 8^{*} \\
41 / 68,60 \cdot 3^{*} \\
6 / 70,8 \cdot 6^{* *} \\
31 / 31,100\end{array}$ & $\begin{array}{r}(11-74) \\
(20-75) \\
(0-22)\end{array}$ & $\begin{array}{r}97 \cdot 0 \\
95 \cdot 1 \\
83 \cdot 3 \\
100\end{array}$ & $\begin{array}{c}- \\
- \\
- \\
38.7\end{array}$ \\
\hline - BSA & $+\mathrm{BSA}$ & + & + & $54 / 69,78 \cdot 3$ & $(64-88)$ & $96 \cdot 3$ & - \\
\hline
\end{tabular}

$\dagger$ Fertilized eggs at telophase-second polar body with sperm heads fully decondensed.

\$ Washed cumulus masses.

${ }^{*} P<0.05,{ }^{* *} P<0.01$ compared with appropriate control group.

When sperm suspensions were preincubated and assessed in the presence of BSA, most of the eggs in all categories were fertilized (Table 1). In contrast, the fertilizing ability of suspensions in BSA-free medium depended on which egg investments were present. With cumulus-intact eggs, whether washed free of oviducal fluid or not, a significantly lower proportion $(P<0.05)$ of eggs was fertilized, compared with BSA-containing controls, and considerable variability between replicates was noted. The introduction of BSA along with cumulus-intact eggs, however, promoted consistently high rates of fertilization. When the cumulus layers were removed, few eggs were fertilized (mean of $8.6 \% ; P<0.01$ compared with control group) but when the zonae were also removed, all eggs were fertilized. The incidence of polyspermy was lower in the BSA-free suspensions and this was examined in a further series of experiments $(\mathrm{N}=5)$. Again, all eggs were fertilized, but the incidence of polyspermy in the BSA-free suspensions $(13 / 71$; mean of $20.2 \%)$ was significantly lower $(P<0.05)$ than that in BSA containing suspensions $(36 / 66$; mean of $55.4 \%)$; this may be a reflection of the lower motility in these samples (discussed below). In all replicates, rapid rates of sperm penetration were observed, with $>80 \%$ of all fertilized eggs at the stage of maximal nuclear development. 
In BSA-containing suspensions, approximately $50-70 \%$ of spermatozoa were motile while in the corresponding BSA-free suspension the proportion was generally about $30-50 \%$. Hyperactivated motility was expressed in about $50 \%$ of motile cells in the presence of BSA and to a lesser extent in its absence $(\sim 20-30 \%)$. Additionally, sperm movements were more erratic and jerky in the absence of BSA. Despite these differences, motility in the BSA-free suspensions was not considered to be so impaired that it alone accounted for the reduced fertilizing ability. The introduction of BSA resulted in increased motility, including hyperactivated motility.

Washed suspensions. In these experiments, suspensions preincubated for $120 \mathrm{~min}$ in BSA-free medium were washed as described above before being assessed. Preliminary investigations revealed that inclusion of a macromolecular component was required to protect the spermatozoa during washing to maintain adequate motility afterwards. Therefore, BSA-free, PVA-containing medium was used $(\mathrm{N}=4)$.

Results obtained with PVA were very similar to those obtained in the absence of a macromolecular component : cumulus-intact eggs, 65/89 (73\%; range 56-93); cumulus-free eggs, $13 / 122(11 \%$; range $0-38)$; zona-free $58 / 58(100 \%)$. Control values were again high with all eggs (data not presented).

Bavister $(1981,1982)$ has reported that the synthetic polymer PVA maintains hamster sperm motility to a level equivalent to that with BSA. In general, subjective assessment of the PVAincubated suspensions indicated that mouse sperm motility was frequently similar to that observed in BSA-containing medium but occasionally it was quantitatively and qualitatively reduced. Considerable variation amongst suspensions incubated in either of the BSA-free media was observed, while the presence of BSA consistently promoted good motility.

\section{Series $I I:$ is albumin required for capacitation?}

Capacitation rate in various concentrations of $B S A$. Sperm suspensions were prepared in media containing BSA at $30,4,1,0.25$ and $0.1 \mathrm{mg} / \mathrm{ml}$. After 30 and $120 \mathrm{~min}$ preincubation, aliquants were removed, diluted in medium of the same composition and assessed for their ability to fertilize cumulus-intact eggs $(\mathrm{N}=2)$.

In the presence of BSA at $30-1 \mathrm{mg} / \mathrm{ml}$, the rate did not differ significantly, with relatively few eggs fertilized after $30 \mathrm{~min}$ preincubation $(30 \mathrm{mg} / \mathrm{ml}: 10 / 40,25 \cdot 0 \% ; 4 \mathrm{mg} / \mathrm{ml}: 14 / 45,31 \cdot 1 \% ; 1$ $\mathrm{mg} / \mathrm{ml}: 11 / 41,26.8 \%$ ) but most eggs were fertilized after $120 \mathrm{~min}$ preincubation $(4 \mathrm{mg} / \mathrm{ml}, 40 / 48$, $83.3 \%$; similar results for the other 2 groups). Few eggs had reached advanced nuclear stages $(<15 \%)$ after $30 \mathrm{~min}$ while the majority had done so by $120 \mathrm{~min}(\mathrm{e} . \mathrm{g} .4 \mathrm{mg} / \mathrm{ml}: 97.5 \%)$. The only detectable differences involved the low concentrations of BSA after a short preincubation, when the proportion of eggs fertilized was significantly lower $(P<0.05)$ than in the control suspension with $4 \mathrm{mg} \mathrm{BSA} / \mathrm{ml}(0.25 \mathrm{mg} / \mathrm{ml}: 3 / 63,4.8 \% ; 0.1 \mathrm{mg} / \mathrm{ml}: 1 / 55,1.8 \%)$. When preincubated for 120 min, these suspensions did not differ significantly from the others examined.

Capacitation rate in the absence of $B S A$. Sperm suspensions were prepared in media with and without BSA; after 30,60, 90 and $120 \mathrm{~min}$, aliquants from both suspensions were diluted in BSAcontaining medium and assessed for their ability to fertilize cumulus-intact eggs $(\mathrm{N}=3)$.

As illustrated in Text-fig. 1, at each time point except the last fewer eggs were fertilized and fewer had reached the stage of maximal nuclear development when the sperm suspensions were preincubated in albumin-free medium. The differences in proportion of eggs fertilized were significant in the 30-min $(P<0.01)$ and 60-min $(P<0.05)$ groups indicating that capacitation did occur in the absence of albumin, but the rate at which these spermatozoa became capacitated lagged behind that of their BSA-incubated counterparts by about $30 \mathrm{~min}$.

\section{Series III: acrosome loss in the presence and absence of albumin}

Three sets of experiments were carried out in this series. In the first, sperm suspensions were prepared in BSA- and PVA-containing media, incubated for $120 \mathrm{~min}$ and diluted 10 -fold. After a 5- 


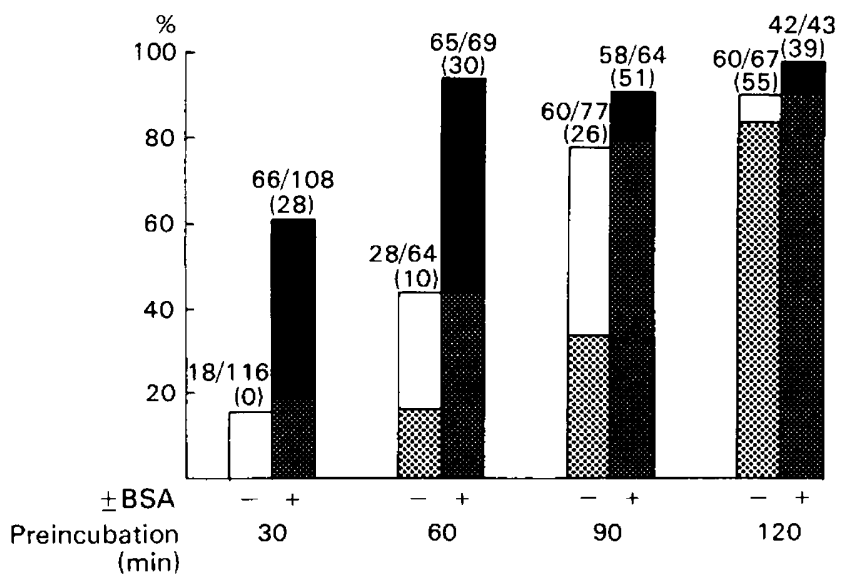

Text-fig. 1. In-vitro fertilizing ability, in the presence of BSA, of mouse spermatozoa preincubated in the absence or presence of BSA. The solid bars represent $\%$ of eggs fertilized and the stippled bars represent \% of eggs with advanced nuclear development. The figures above each bar indicate the number of eggs fertilized/total eggs and the figures in parentheses indicate the number of eggs with advanced nuclear development.

min incubation, samples were filtered and assessed. The following groups were examined: BSAincubated $\rightarrow$ BSA; PVA $\rightarrow$ PVA; PVA $\rightarrow$ BSA; PVA (washed at $120 \mathrm{~min}) \rightarrow$ PVA $(\mathrm{N}=3)$. The results of these experiments indicated that acrosome loss was significantly lower $(P<0.05)$ when suspensions were incubated in the presence of PVA (14.7\%), but within 5 min of BSA introduction the values $(28.0 \%)$ did not differ significantly from those observed in the suspensions continuously exposed to BSA $(37.7 \%)$. Washing the PVA-incubated samples stimulated acrosome loss even more effectively $(49.0 \% ; P<0.01$ compared with PVA $\rightarrow$ PVA). This loss was also significantly higher $(P<0.05)$ than that seen in the BSA $\rightarrow$ BSA group, but the considerable variability in the washed samples probably makes this of limited biological relevance. These assessments also indicate that the low incidence of acrosome loss in unwashed PVA suspensions was not an artefact of filtration in the presence of PVA rather than BSA.

Having established that introduction of BSA stimulated acrosome loss in PVA-incubated spermatozoa, the second set of experiments $(N=3)$ examined the rate of acrosome loss in the presence of fatty acid-free BSA (faf BSA) compared with the crystalline BSA routinely used. Sperm suspensions, using the same males, were prepared in BSA, faf BSA and PVA. After 30-min incubation, aliquants were diluted and assessed as above : BSA $\rightarrow$ BSA; BSA $\rightarrow$ faf BSA ; faf BSA $\rightarrow$ faf BSA. PVA suspensions were not examined at this time. The original suspensions were incubated for another $90 \mathrm{~min}$ and the following were assessed: BSA $\rightarrow$ BSA; BSA $\rightarrow$ faf BSA; faf BSA $\rightarrow$ faf BSA; PVA $\rightarrow$ PVA; PVA $\rightarrow$ BSA; PVA $\rightarrow$ faf BSA. The results from these experiments are shown in Table 2. Acrosome loss at $30 \mathrm{~min}$ was low $(<10 \%)$, whether spermatozoa were incubated in BSA or faf BSA, but the values had risen significantly $(P<0.01)$ in all groups incubated for $120 \mathrm{~min}$ in the presence of albumin (mean values $\sim 35-40 \%$ ). There were no differences in acrosome loss between the BSA- and faf BSA-incubated suspensions. As in the first set of experiments, acrosome loss in the PVA-incubated suspensions was low $(\sim 11 \%)$, but upon the introduction of albumin these values rose to a level $(\sim 28 \%)$ that did not differ significantly from the control samples. Again, no differences between the 2 albumin preparations were noted.

In a third set of experiments $(\mathrm{N}=3)$, the response of spermatozoa, incubated for $2 \mathrm{~h}$ in PVA, to the introduction of various concentrations of BSA $(30,4,1,0.25$ and $0.1 \mathrm{mg} / \mathrm{ml})$ was examined. In addition, a sample of BSA (prepared by R. A. P. Harrison) which had been defatted on a Dowex 1X8 column, further purified on a Con A .Jepharose column to remove glycoprotein contaminants 
Table 2. Acrosome loss in mouse sperm suspensions incubated in the presence of BSA (crystalline or fatty acid-free) or PVA

\begin{tabular}{|c|c|c|c|}
\hline \multirow{2}{*}{$\begin{array}{l}\text { Preincubation time } \\
\text { (min) }\end{array}$} & \multicolumn{2}{|c|}{ Macromolecular component } & \multirow{2}{*}{$\begin{array}{c}\% \text { Spermatozoa lacking } \\
\text { acrosome } \\
\text { (mean } \pm \text { s.e.m. })\end{array}$} \\
\hline & Preincubation & Dilution & \\
\hline \multirow[t]{2}{*}{30} & BSA & $\begin{array}{c}\text { BSA } \\
\text { faf BSA }\end{array}$ & $\begin{array}{l}4.3 \pm 1.4 \ddagger \\
6.7 \pm 0.9\end{array}$ \\
\hline & faf BSA & faf BSA & $4.7 \pm 0.9$ \\
\hline \multirow[t]{3}{*}{120} & BSA & $\begin{array}{c}\text { BSA } \\
\text { faf BSA }\end{array}$ & $\begin{array}{l}34 \cdot 3 \pm 2 \cdot 4 \ddagger^{\dagger} \\
39 \cdot 7 \pm 4 \cdot 1\end{array}$ \\
\hline & faf BSA & faf BSA & $34.0 \pm 6.9$ \\
\hline & PVA & $\begin{array}{c}\text { PVA } \\
\text { BSA } \\
\text { faf BSA }\end{array}$ & $\begin{array}{l}10 \cdot 7 \pm 0 \cdot 7^{*}+ \\
27 \cdot 3 \pm 0 \cdot 9^{*} \\
28 \cdot 7 \pm 2 \cdot 2\end{array}$ \\
\hline
\end{tabular}

$* P<0.05, \dagger P<0.01, \ddagger P<0.01$.

Table 3. Acrosome loss in mouse sperm suspensions incubated for $2 \mathrm{~h}$ in the presence of PVA at 1 or $4 \mathrm{mg} / \mathrm{ml}$ and then diluted into medium containing BSA at $0 \cdot 1-30 \mathrm{mg} / \mathrm{ml}$

\begin{tabular}{|c|c|c|c|}
\hline \multicolumn{2}{|c|}{ Macromolecular component } & \multirow{2}{*}{$\begin{array}{l}\text { Conc. } \\
(\mathrm{mg} / \mathrm{ml})\end{array}$} & \multirow{2}{*}{$\begin{array}{c}\% \text { Spermatozoa lacking } \\
\text { acrosome } \\
\text { (mean } \pm \text { s.e.m.) }\end{array}$} \\
\hline Incubation & Dilution & & \\
\hline \multirow[t]{7}{*}{ PVA } & PVA & 1 & $4.8 \pm 0.7$ \\
\hline & BSA & 30 & $20.5 \pm 4 \cdot 5^{*}$ \\
\hline & & 4 & $23.3 \pm 3.3^{*}$ \\
\hline & & 1 & $19.0 \pm 3.7^{*}$ \\
\hline & & $1 \dagger$ & $23.0 \pm 5 \cdot 0^{*}$ \\
\hline & & 0.25 & $19.7 \pm 3.2^{*}$ \\
\hline & & $0 \cdot 1$ & $20.0 \pm 2 \cdot 1^{*}$ \\
\hline PVA & PVA & 4 & $5.5+0.5$ \\
\hline
\end{tabular}

$\dagger$ Purified BSA; see text for details.

$* P<0.05$ compared with PVA.

and finally subjected to gel filtration on Ultrogel AcA 44 was used at $1 \mathrm{mg} / \mathrm{ml}$. Acrosome loss in the presence of PVA alone was low, but the introduction of BSA at all concentrations from 0.1 to 30 $\mathrm{mg} / \mathrm{ml}$ triggered a significantly higher $(P<0.05)$ incidence of acrosome loss; the purified BSA was equally effective (Table 3). Finally, incubation in medium containing $4 \mathrm{mg} \mathrm{PVA} / \mathrm{ml} \mathrm{did} \mathrm{not}$ stimulate acrosome loss above that observed in the $1 \mathrm{mg} \mathrm{PVA} / \mathrm{ml}$ used in all other experiments.

\section{Series IV: effects on fertilization of washing sperm suspensions}

Because washing uncapacitated sperm suspensions can markedly increase their fertilizing ability (Fraser, 1984), suspensions were prepared in media with and without BSA, preincubated for $30 \mathrm{~min}$ and aliquants from each sample were washed. Washed and unwashed 30 -min suspensions were diluted in BSA-containing medium and assessed for their ability to fertilize cumulus-intact eggs. Suspensions in BSA-containing medium were preincubated further and assessed at $120 \mathrm{~min}$ $(\mathrm{N}=4)$. In 2 of these replicates, fatty acid-free BSA (faf BSA) was also used to determine whether capacitation was accelerated, relative to that with the crystalline BSA used throughout these experiments. 
Sperm suspensions washed after 30 -min preincubation in both media were significantly more fertile $(P<0.01$; Table 4$)$ than their unwashed counterparts. While suspensions preincubated in the presence of BSA and then washed were indistinguishable from the 120 -min unwashed samples, those in the washed, BSA-free group were less fertile $(P<0.05)$ than the unwashed 120 -min control suspensions. An even more striking difference in rate of sperm penetration was noted, with few fertilizing eggs having reached the stage of maximal nuclear development within the $75 \mathrm{~min}$ allowed $(9 \cdot 4 \%$ compared with $96.6 \%$ for 30 -min + BSA washed and $94.2 \%$ for 120 -min + BSA unwashed samples). Thus once these spermatozoa, preincubated in the absence of BSA and washed, were exposed to BSA they became fertile but at a slower rate.

Results from the 2 suspensions preincubated in faf BSA did not differ from their counterparts in the standard BSA: faf BSA, unwashed-20/33 (61\%) fertilized with $55 \%$ at maximal nuclear development; faf BSA, washed-25/27 (93\%) fertilized with $92 \%$ fully developed; BSA, unwashed-15/31 (50\%) fertilized with 53\% fully developed; BSA, washed-33/34 (97\%) fertilized with $97 \%$ fully developed.

Table 4. In-vitro fertilizing ability of mouse sperm suspensions before and after washing to remove inhibitory factor

\begin{tabular}{|c|c|c|c|c|c|}
\hline \multicolumn{2}{|c|}{ Preincubation } & \multirow[b]{2}{*}{ \pm Washing } & \multicolumn{2}{|c|}{ Eggs fertilized $\dagger$} & \multirow{2}{*}{$\begin{array}{l}\text { Maximal nuclear } \\
\text { development, \% }\end{array}$} \\
\hline Time (min) & Medium & & No., $\%$ & (range) & \\
\hline \multirow[t]{2}{*}{30} & $-\mathrm{BSA}$ & $\overline{+}$ & $\begin{array}{c}3 / 60, \quad 5 \cdot 0 \\
64 / 88,72 \cdot 7^{* *}\end{array}$ & $\begin{array}{c}(0-30) \\
(59-100)\end{array}$ & $\begin{array}{r}0 \\
9.4\end{array}$ \\
\hline & $+\mathrm{BSA}$ & - & $\begin{array}{l}21 / 76,27 \cdot 6 \\
58 / 63,92 \cdot 1^{* *}\end{array}$ & $\begin{array}{c}(0-56) \\
(82-100)\end{array}$ & $\begin{array}{l}38 \cdot 1 \\
96 \cdot 6\end{array}$ \\
\hline 120 & $+\mathrm{BSA}$ & - & $69 / 72,95.8$ & $(90-100)$ & 94.2 \\
\hline
\end{tabular}

† BSA was present in all suspensions during fertilization.

** $P<0.01$ compared with value for no washing.

Negligible levels of hyperactivated motility were observed in all 30-min unwashed suspensions. After washing, this pattern of motility was observed in $15-30 \%$ of single motile cells in suspensions preincubated in BSA (including faf BSA), but not those prepared in BSA-free medium. This delay in onset of hyperactivated motility after washing correlates well with the delayed penetration exhibited by these spermatozoa.

\section{Series $V$ : inhibitory effects of sperm supernatants}

The washing procedure used above removes a surface-associated inhibitory component from mouse spermatozoa (Fraser, 1984). Since the results in Series IV suggested that inhibitor removal was less efficient in BSA-free medium, this was examined directly by preparing sperm supernatants from suspensions incubated in the presence and absence of BSA and assessing the extent to which these inhibited the fertilizing ability of capacitated suspensions (preincubated $120 \mathrm{~min}$ in the presence of BSA). Three dilutions $(1 / 2,1 / 5$ and $1 / 10)$ were assessed. BSA $(4 \mathrm{mg} / \mathrm{ml})$ was added to all BSA-free supernatants before use $(\mathrm{N}=2)$.

The control, capacitated suspensions were highly fertile $(94 \cdot 1 \%$ of eggs fertilized; Table 5$)$. The introduction of sperm supernatant at a $1 / 2$ dilution resulted in significant reductions in fertility (+ BSA supernatant, $P<0.01 ;-$ BSA supernatant, $P<0.05$ ). Using higher dilutions, only the supernatants from BSA-containing suspensions were inhibitory at $1 / 5$; neither group was inhibitory at $1 / 10$. The inhibitory effect was therefore more pronounced in the + BSA supernatants. 
Table 5. Inhibition of capacitated mouse sperm fertilizing ability in vitro by a factor in epididymal sperm suspensions preincubated for $30 \mathrm{~min}$ in the presence or absence of BSA

\begin{tabular}{|c|c|c|c|c|c|}
\hline \multirow{2}{*}{$\begin{array}{l}\text { Fertilization } \\
\text { medium }\end{array}$} & \multirow{2}{*}{$\begin{array}{l}\text { Supernatant } \\
\text { dilution factor }\end{array}$} & \multirow{2}{*}{$\begin{array}{c}\text { Preincubation } \\
\pm \text { BSA }\end{array}$} & \multicolumn{2}{|c|}{ Eggs fertilized $\dagger$} & \multirow{2}{*}{$\begin{array}{l}\text { Maximal nuclear } \\
\text { development, \% }\end{array}$} \\
\hline & & & No., \% & (range) & \\
\hline \multirow[t]{3}{*}{$\begin{array}{l}\text { Control } \\
\text { Sperm } \\
\text { supernatant }\end{array}$} & $\overline{1 / 2}$ & $\begin{array}{l}+ \\
+ \\
-\end{array}$ & $\begin{array}{c}48 / 51,94 \cdot 1 \\
1 / 25,4 \cdot 0^{* *} \\
13 / 35,37 \cdot 1^{*}\end{array}$ & $\begin{array}{c}(87-100) \\
(0-9) \\
(31-40)\end{array}$ & $\begin{array}{r}100 \\
0 \\
69 \cdot 2\end{array}$ \\
\hline & $1 / 5$ & $\begin{array}{l}+ \\
-\end{array}$ & $\begin{array}{c}8 / 31,25 \cdot 8^{*} \\
28 / 33,84 \cdot 8\end{array}$ & $\begin{array}{l}(18-30) \\
(79-100)\end{array}$ & $\begin{array}{l}87 \cdot 5 \\
92 \cdot 3\end{array}$ \\
\hline & $1 / 10$ & $\begin{array}{l}+ \\
-\end{array}$ & $\begin{array}{l}36 / 51,70 \cdot 6 \\
24 / 33,72 \cdot 7\end{array}$ & $\begin{array}{l}(67-73) \\
(71-75)\end{array}$ & $\begin{array}{l}75 \cdot 0 \\
87 \cdot 5\end{array}$ \\
\hline
\end{tabular}

† BSA was present in all suspensions during fertilization.

${ }^{*} P<0.05,{ }^{* *} P<0.01$ compared with control values.

\section{Series VI: hyperosmolal treatment in the absence of albumin}

In the final series of experiments, sperm suspensions were prepared in 4 different media by releasing half the contents of 1 epididymis from each of 2 males into $0.5 \mathrm{ml}$ of : + BSA, $318 \mathrm{mosmol}$; - BSA, 318 mosmol; - BSA, $368 \mathrm{mosmol}$; - BSA, 418 mosmol. At $5 \mathrm{~min}$ after release, all suspensions were washed and then diluted in BSA-containing medium. The ability of the spermatozoa to fertilize cumulus-intact eggs was then assessed $(\mathrm{N}=3)$.

Table 6. In-vitro fertilizing ability of sperm suspensions prepared in isoosmolal and hyperosmolal media with and without BSA, incubated $5 \mathrm{~min}$ and then washed to remove inhibitory factor

\begin{tabular}{lllc}
\hline & \multicolumn{2}{c}{ Eggs fertilized $\dagger$} & \multicolumn{1}{c}{$\begin{array}{c}\text { Maximal nuclear } \\
\text { development, \% }\end{array}$} \\
\cline { 2 - 3 } \multicolumn{1}{c}{ Medium for release } & \multicolumn{1}{c}{ No., \% } & (range) & $28 \cdot 6$ \\
\hline Control (+ BSA, 318 mosmol) & $42 / 52,80 \cdot 8$ & $(33-100)$ & $5 \cdot 9$ \\
- BSA (318 mosmol) & $17 / 39,43 \cdot 6^{*}$ & $(39-50)$ & $40 \cdot 1$ \\
- BSA (368 mosmol) & $49 / 60,81 \cdot 7$ & $(75-94)$ & $30 \cdot 3$ \\
- BSA (418 mosmol) & $33 / 47,70 \cdot 2$ & $(67-77)$ & \\
\hline
\end{tabular}

† BSA was present in all suspensions during fertilization.

${ }^{*} P<0.05$ compared with control.

As noted in Series IV, spermatozoa released into and preincubated (albeit for a shorter time) in iso-osmolal BSA-free medium were less fertile $(P<0.05$; Table 6) after washing and the introduction of BSA than those released into BSA-containing medium. By increasing the osmolality of the BSA-free medium to levels previously demonstrated to enhance fertilizing ability (Fraser, 1983b), washing and introduction of BSA rendered these suspensions indistinguishable from the control samples. In all groups, the rate of penetration was slower than observed in fully capacitated suspensions (e.g. Tables $1,4,5)$.

\section{Discussion}

The results obtained in the present study indicate that albumin is required for mouse spermatozoa to achieve efficient fertilization of zona-intact eggs (Table 1), thus confirming earlier studies (Miyamoto \& Chang, 1973; Hoppe \& Whitten, 1974; Quinn et al., 1982). The fact that the majority 
of cumulus-intact eggs was fertilized, even in albumin-free medium, suggests that sufficient albumin or similar macromolecule is present in the cumulus masses, possibly in residual oviducal and/or follicular fluid trapped amongst the cumulus cell complex, to promote sperm penetration. Analyses of these fluids from many species have revealed the presence of albumin (e.g. Mastroianni, Urzua \& Stambaugh, 1970; Lippes, Enders, Praday \& Bartholomew, 1972; Edwards, 1974; Lui et al., 1977). Given the range of results obtained (see Table 1), however, the amount or accessibility of the effector appears to be variable. A much more uniform response was obtained when a fixed concentration of albumin was introduced with the eggs (Table 1; Text-fig. 1). Despite the extensive manipulation during hyaluronidase treatment to remove cumulus cells, which should have removed follicular and oviducal fluids as well, a few cumulus-free eggs were fertilized in albumin-free medium. This was not due to small amounts of protein derived from epididymal fluid or released during sperm preincubation (as suggested by Quinn et al., 1982), since suspensions washed before assessment showed the same low levels of fertilizing ability (Series I). It would appear that at least the occasional mouse spermatozoon can penetrate the zona in the absence of exogenous albumin.

The fact that albumin-free media supported fertilization of all zona-free eggs, albeit with a lower incidence of polyspermy, is at variance with the study of Quinn et al. (1982) who reported no fertilization of such eggs in BSA-free, polyvinyl pyrrolidone-containing medium. However, fertilization of all eggs was consistently obtained in the present study under a variety of BSA-free conditions ( \pm washing of sperm suspensions, \pm PVA), and this would indicate that the results are neither spurious nor artefactual. Indeed, Barros, Berrios \& Herrera (1973) reported the fertilization of zona-free hamster eggs by guinea-pig spermatozoa in an albumin-free medium, a finding consistent with the present study. Perhaps the polyvinyl pyrrolidone used in the earlier study contained toxic material of low molecular weight (see Bavister, 1974) which prevented normal sperm-egg plasma membrane interaction.

A requirement for albumin at fertilization does not necessarily imply a requirement for capacitation per se, the time-dependent transformation of a non-fertilizing spermatozoon into a potentially fertilizing one (Bedford, 1970). In fact, mouse spermatozoa can undergo capacitation in the absence of exogenously supplied macromolecules, although the rate of capacitation lags about $30 \mathrm{~min}$ behind that observed in BSA-containing medium (Text-fig. 1). Once capacitated, the introduction of BSA promotes rapid sperm penetration. The rate of capacitation in media containing 1-30 mg BSA $/ \mathrm{ml}$ is similar, with no evidence for improved or accelerated capacitation in high concentrations of BSA as reported by Miyamoto \& Chang (1973). Capacitation is rather slower in low concentrations of BSA $(0.25$ and $0.1 \mathrm{mg} / \mathrm{ml})$, but after preincubation for $120 \mathrm{~min}$ all suspensions are highly fertile.

The failure of spermatozoa to fertilize zona-intact eggs in the absence of BSA could be due to faulty motility and/or failure to undergo the acrosome reaction. Certainly many of the BSA-free suspensions differed quantitatively and qualitatively from their counterparts incubated in the presence of BSA, with fewer motile cells and fewer exhibiting the hyperactivated motility which is specifically required for zona penetration (Fraser, 1981), although the introduction of BSA improved both characteristics. Few of these suspensions were considered to be extremely poor, however, and the inclusion of PVA had a positive effect on maintenance of motility. A more precise response, or lack thereof, was observed when acrosome loss was assessed. In spermatozoa incubated for $120 \mathrm{~min}$ in the absence of BSA, acrosome loss was very low but within 5 min of BSA introduction (using concentrations ranging from 0.1 to $30 \mathrm{mg} / \mathrm{ml}$ ) it was stimulated to the level seen in cells maintained continuously in the presence of BSA. Albumin therefore promotes the acrosome reaction in capacitated spermatozoa, confirming the original observation of Lui et al. (1977) that albumin is required for the acrosome reaction to occur. The lag in sperm penetration when albumin was introduced after shorter preincubations indicates that uncapacitated and partly capacitated spermatozoa cannot immediately respond in this manner, an observation consistent with the low levels of acrosome loss seen in uncapacitated $(30 \mathrm{~min})$ suspensions incubated in BSA-containing 
media (Table 2). Since the introduction of albumin both triggers the acrosome reaction and enhances motility, the ability of egg-cumulus complex components to support fertilization (Series I; Bavister, 1982) suggests that albumin is a major, if not the sole, molecular species involved.

Under permissive conditions, acrosome loss in mouse sperm populations increases with time (e.g. present study; Fraser, 1981). It would therefore appear that the introduction of albumin triggers the acrosome reaction in those cells that otherwise would have lost the acrosome during the incubation phase. A similar response was noted when glucose was introduced to suspensions capacitated in glucose-free medium (Fraser \& Quinn, 1981). Whether the fertilizing spermatozoa are drawn from this pool, however, or from the acrosome-intact cells with the potential to undergo the acrosome reaction is unclear. The latter is supported by the observed failure of washed, albumin-free spermatozoa to penetrate the zona pellucida (Series I), despite the demonstrable and significant increase in acrosome loss, compared with unwashed samples. If the spermatozoa which fertilize zona-free eggs also come from this population, then direct contact with the egg's surface is able to promote acrosome loss since only acrosome-reacted spermatozoa appear capable of fertilizing such eggs (Yanagimachi, 1981). A similar suggestion was made by Fraser (1983a) based on the high fertility and high incidence of polyspermy obtained with sperm populations exhibiting a low incidence of overt acrosome loss (preincubated for $30 \mathrm{~min}$ in standard media).

Given that the secretions of both the male and female tracts contain albumin, it seems unlikely that, under normal conditions, albumin actually triggers the acrosome reaction; rather, it would provide a permissive environment in which the acrosome reaction might occur. The specific stimulus, at least in vivo, would appear to be associated with the ampulla and/or the eggs and its investments since appreciable acrosome loss in vivo is observed only in the immediate vicinity of eggs (Cummins \& Yanagimachi, 1982). It has been proposed that the zona pellucida specifically initiates the acrosome reaction in the fertilizing spermatozoon (e.g. Saling, Sowinski \& Storey, 1979), although this has not met with universal acceptance (Bedford, 1983). The present results indicate that the zona, in the absence of albumin, is unable to promote acrosome loss and subsequent fertilization. It is not possible to speculate whether the fertilization achieved with cumulus-intact eggs in albumin-free media represents a permissive environment provided by the cumulus complex plus a specific stimulus from the zona, or whether the cumulus complex alone initiated the acrosome reaction.

Fraser (1984) has presented evidence that capacitation of mouse spermatozoa involves the loss of a surface-associated inhibitory component. Removal of the component from uncapacitated spermatozoa by washing immediately and significantly increases sperm fertilizing ability, while addition of sperm supernatants to capacitated suspensions significantly reduces fertilizing ability. The current study provides evidence that albumin aids or enhances loss of the inhibitory component. In the absence of exogenous albumin, this loss occurs more slowly which would account for the slower rate of capacitation, the less marked response to washing and the corresponding lower levels of inhibition obtained with supernatants under albumin-free conditions. Aonuma, Okabe, Kishi, Kawaguchi \& Yamada (1982) have also reported that less inhibitory activity is released during washing in albumin-free medium, although they failed to identify a specific mechanism of action. Fraser (1984) demonstrated that introduction of the inhibitor to capacitated spermatozoa effectively arrested acrosome loss, suggesting that the presence of inhibitor stabilizes the membranes and thereby prevents the acrosome reaction. The present study has shown that the final steps preceding the acrosome reaction do not occur in the absence of albumin. The fact that either washing or introducing albumin immediately triggers the acrosome reaction in capacitated spermatozoa suggests that some final removal of the inhibitor is directly linked with the membrane fusion events of the reaction. The enhanced fertilizing ability of sperm suspensions exposed to hyperosmolal media, conditions which would promote the loss of surface-associated compounds, before washing and introduction of BSA is consistent with this proposed mechanism of action.

Several current theories of the molecular mechanisms involved in the acrosome reaction include 
a role for albumin, aiding removal of molecules attached to the sperm surface and of free fatty acids released by sperm phospholipase activity (reviewed by Yanagimachi, 1981; Harrison, 1983; Meizel, 1984). The present study provides further evidence for the first mechanism and would be consistent with the second. Commercially available albumin is a complex macromolecule with numerous adsorbed components including fatty acids. The possible importance of the fatty acid content of BSA during interactions with spermatozoa has been highlighted by observations that fatty acid-saturated BSA can inhibit acrosome loss (hamster: Lui \& Meizel, 1977) and fertilizing ability (mouse: Quinn \& Whittingham, 1982). Results obtained with fatty acid-free BSA are less consistent, with reports of enhanced acrosome loss in hamster spermatozoa (Lui \& Meizel, 1977) and fertilizing ability in rat spermatozoa (Davis, 1976) while the present study could detect no differences between crystalline BSA (similar to that used by Lui \& Meizel, 1977) and fatty acid-free BSA in either the rate of capacitation (Series IV) or the incidence of acrosome loss in uncapacitated and capacitated suspensions. Furthermore, these two preparations were indistinguishable in their ability to induce acrosome loss in spermatozoa capacitated in the absence of BSA.

The fatty acid content of the BSA sample used in the present work was not determined, but in an extensive evaluation of crystalline and Fraction V albumin samples, Chen (1967) found values ranging from 0.06 to $2.5 \mathrm{~mol}$ fatty acid per mol albumin. Given that albumin has 7 binding sites for fatty acids (Reed, 1977), commercially available albumins are generally not saturated. It is possible, however, that the apparent discrepancies reported for unpurified and fatty acid-free albumins reflect variability as observed by Chen (1967), with some unpurified samples having an unusually high fatty acid content.

Since most albumins would have unfilled binding sites for fatty acids, the mechanisms whereby albumin can promote the acrosome reaction in capacitated spermatozoa may well include the removal of lipids from the sperm surface. While the suggestion has been made that other molecules, in particular phospholipase, present in unpurified albumin preparations may be responsible for albumin-associated phenomena (discussed by Meizel, 1984), a highly purified albumin preparation was able to stimulate acrosome loss to the same extent as less purified albumin (Series III). This albumin is also effective in promoting the removal of the surface inhibitory component (L. R. Fraser, unpublished results). These data, therefore, do not provide support for the above proposal.

In conclusion, albumin is required for the capacitated mouse spermatozoon to undergo the acrosome reaction although capacitation itself can occur in the absence of albumin. Albumin promotes the loss of a surface-associated inhibitory component which stabilizes sperm membranes and it also plays a more general role in maintaining sperm motility, including hyperactivated motility.

I thank Dr. Robin Harrison for many stimulating discussions and for provision of the purified BSA. This work was supported in part by a grant from the AFRC.

\section{References}

Aonuma, S., Okabe, M., Kishi, Y., Kawaguchi, M. \& Yamada, H. (1982) Capacitation inducing activity of serum albumin in fertilization of mouse ova in vitro. $J$. Pharm. Dyn. 5, 980-987.

Barros, C., Berrios, M. \& Herrera, E. (1973) Capacitation in vitro of guinea-pig spermatozoa in a saline solution. J. Reprod. Fert. 34, 547-549.

Bavister, B.D. (1974) The effect of variations in culture conditions on the motility of hamster spermatozoa. $J$. Reprod. Fert. 38, 431-440.

Bavister, B.D. (1981) Substitution of a synthetic polymer for protein in a mammalian gamete culture system. $J$. exp. Zool. 217, 45-51.
Bavister, B.D. (1982) Evidence for a role of postovulatory cumulus components in supporting fertilizing ability of hamster spermatozoa. J. Androl. 3, 365372.

Bavister, B.D. \& Yanagimachi, R. (1977) The effects of sperm extracts and energy sources on the motility and acrosome reaction of hamster spermatozoa in vitro. Biol. Reprod. 16, 228-237.

Bedford, J.M. (1970) Sperm capacitation and fertilization in mammals. Biol. Reprod., Suppl. 2, 128-158.

Bedford, J.M. (1983) Significance of the need for sperm capacitation before fertilization in eutherian mammals. Biol. Reprod. 28, 108-120. 
Chen, R.F. (1967) Removal of fatty acids from serum albumin by charcoal treatment. J. biol. Chem. 242, 173-181.

Cummins, J.M. \& Yanagimachi, R. (1982) Sperm-egg ratios and the site of the acrosome reaction during in vivo fertilization in the hamster. Gamete Res. 5, 239 . 256.

Davis, B.K. (1976) Influence of serum albumin on the fertilizing ability in vitro of rat spermatozoa. Proc. Soc. exp. Biol. Med. 151, 240-243.

Edwards, R.G. (1974) Follicular fluid. J. Reprod. Fert. 37, 189-219.

Fraser, L.R. (1981) Dibutyryl cyclic AMP decreases capacitation time in vitro in mouse spermatozoa. $J$. Reprod. Fert. 62, 63-72.

Fraser, L.R. (1983a) Mouse sperm capacitation assessed by kinetics and morphology of fertilization in vitro. $J$. Reprod. Fert. 69, 419-428.

Fraser, L.R. (1983b) Potassium ions modulate expression of mouse sperm fertilizing ability, acrosome reaction and hyperactivated motility in citro. J. Reprod. Fert. 69, 539-553.

Fraser, L.R. (1984) Mouse sperm capacitation in vitro involves loss of a surface-associated inhibitory component. J. Reprod. Fert. 72, 373-384.

Fraser, L.R. \& Quinn, P.J. (1981) A glycolytic product is obligatory for initiation of the sperm acrosome reaction and whiplash motility required for fertilization in the mouse. J. Reprod. Fert. 61, 25-35.

Harrison, R.A.P. (1983) The acrosome, its hydrolases, and egg penetration. In The Sperm Cell, pp. 259-273. Ed. J. André. Martinus Nijhoff, The Hague.

Harrison, R.A.P., Dott, H.M. \& Foster, G.C. (1982) Bovine serum albumin, sperm motility, and the 'dilution effect'. J. exp. Zool. 222, 81-88.

Hoppe, P.C. \& Whitten, W.K. (1974) An albumin requirement for fertilization of mouse eggs in vitro. $J$. Reprod. Fert. 39, 433-436.

Lippes, 3., Enders, R.G., Praday, D.A. \& Bartholomew, W.R. (1972) The collection and analysis of human Fallopian tubal fluid. Contraception 5, 85-92.
Lui, C.W. \& Meizel, S. (1977) Biochemical studies of the in vitro acrosome reaction inducing activity of bovine serum albumin. Differentiation 9, 59-66.

Lui, C.W., Cornett, L.W. \& Meizel, S. (1977) Identification of the bovine follicular protein involved in the in vitro induction of the hamster sperm acrosome reaction. Biol. Reprod. 17, 34-41.

Mastroianni, L., Urzua, M. \& Stambaugh, R. (1970) Protein patterns in monkey oviductal fluid before and after ovulation. Fert. Steril. 21, 817-820.

Meizel, S. (1984) The importance of hydrolytic enzymes to an exocytotic event, the mammalian sperm acrosome reaction. Biol. Rev. 59, 125-157.

Miyamoto, H. \& Chang, M.C. (1973) The importance of serum albumin and metabolic intermediates for capacitation of spermatozoa and fertilization of mouse eggs in vitro. J. Reprod. Fert. 32, 193-205.

Quinn, P. \& Whittingham, D.G. (1982) Effect of fatty acids on fertilization and development of mouse embryos in vitro. J. Androl. 3, 440-444.

Quinn, P., Stanger, J.D. \& Whittingham, D.G. (1982) Effect of albumin on fertilization of mouse ova in vitro. Gamete Res. 6, 305-313.

Reed, R.G. (1977) Kinetics of bilirubin binding to bovine serum albumin and the effects of palmitate. J. biol. Chem. 252, 7483-7487.

Saling, P.M., Sowinski, J. \& Storey, B.T. (1979) An ultrastructural study of epididymal mouse spermatozoa binding to zonae pellucidae in vitro: Sequential relationship to the acrosome reaction. J. exp. Zool. 209, 229-238.

Snedecor, G. \& Cochran, W. (1967) Statistical Methods, 6th edn. Iowa State University Press, Ames.

Suter, D., Chow, P.Y.W. \& Martin, I.C.A. (1979) Maintenance of motility in human spermatozoa by energy derived through oxidative phosphorylation and addition of albumin. Biol. Reprod. 20, 505-510.

Yanagimachi, R. (1981) Mechanisms of fertilization in mammals. In Fertilization and Embryonic Development in vitro, pp. 81-182. Eds L. Mastroianni \& J. D. Biggers. Plenum Publishing, New York. 